\title{
Research in brief: Colchicine for chronic coronary disease: a recent randomised controlled trial
}

\author{
Authors: Rajan S Pooni ${ }^{A}$ and Tevfik F Ismail ${ }^{B}$
}

DOI: $10.7861 /$ clinmed.2020-0981

\section{Background}

Summary of Nidorf SM, Fiolet ATL, Mosterd A et al. Colchicine in patients with chronic coronary disease. N Engl J Med 2020;383:1838-47.

Atherosclerosis and its sequelae remain the leading cause of cardiovascular-related mortality worldwide. ${ }^{2-4}$ A combination of genetic and acquired risk factors contribute to an inflammatory milieu that leads to atherosclerotic plaque formation and ultimately plaque rupture. ${ }^{5,6}$ Given the central role of inflammation in this process, recent efforts to improve outcomes for patients with coronary disease have focused on targeting inflammatory pathways.

The selective IL-1 $\beta$ inhibitor, canakinumab, has been shown to reduce cardiovascular events among high-risk patients with a history of myocardial infarction (MI) and elevated high-sensitivity C-reactive protein. ${ }^{7}$ Similarly, low-dose colchicine, an agent with a broader spectrum of anti-inflammatory properties, has also shown benefit in post-infarct patients. ${ }^{8,9}$ However, the impact of such an approach in patients with otherwise stable chronic coronary disease was hitherto unknown. Here, we review a recent multi-centre randomised controlled trial (LoDoCo2) evaluating the efficacy of low-dose colchicine in this population. ${ }^{1}$

\section{Study review}

Following an initial open-label phase in which all 6,528 patients enrolled in the study received low-dose colchicine ( $500 \mu \mathrm{g}$ daily), a total of 5,522 patients underwent randomisation; 2,762 were assigned to the colchicine group and 2,760 to the placebo group. Key inclusion criteria included established coronary disease diagnosed either on invasive coronary angiography or computed tomography coronary angiography (coronary artery calcium $\geq 400 \mathrm{HU}$ ), and clinical stability for at least 6 months prior to enrolment. This contrasts with previous studies utilising colchicine that recruited patients within 30 days of an acute MI or were open-label/non-blinded throughout. ${ }^{7,8}$ However, a large number of patients in LoDoCo2 had been subjected to coronary angiography because of previous acute coronary syndromes (84\%), albeit at least 6 months prior to enrolment.

The primary endpoint was a composite of cardiovascular outcomes including cardiovascular mortality, MI, ischaemic stroke and unplanned coronary intervention. The median duration of follow-up across both groups was 28.6 months. The primary endpoint was reached by $6.8 \%$ of patients in the intervention

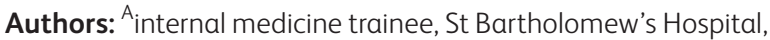
London, UK; ${ }^{\text {' }}$ clinical senior lecturer, King's College London, London, UK and consultant cardiologist, Guy's and St Thomas' NHS Foundation Trust, London, UK group compared to $9.6 \%$ in the control group (intention-to-treat analysis, number needed to treat $\sim 36$ ).

Despite the observation of a statistically significant benefit of colchicine when compared with placebo, there are several study limitations to consider. During the initial open-label phase, a significant proportion of patients (15.7\%) dropped out prior to subsequent randomisation, shifting the paradigm from intentionto-treat towards as-treated analysis by excluding a proportion of suitable patients who could not tolerate the therapy prior to formal data interpretation. Furthermore, this phase confounds the results of the study through administration of colchicine to all patients prior to randomisation. Of note however, after randomisation, the premature discontinuation rate was exactly $10.5 \%$ in both treatment and placebo arms.

Although the study was powered to detect a $30 \%$ reduction in events with colchicine, the actual definition of the primary endpoint was changed several times during the study, presumably to ensure that an adequate number of events were accrued. Furthermore, with $84 \%$ of patients having a prior history of acute coronary syndrome at least 6 months prior to enrolment, and the event rate being driven by ischaemia-related revascularisation, the magnitude of benefit in lower risk populations remains unclear. Finally, a higher, albeit non-significant, incidence of noncardiovascular-related mortality was observed in the colchicine group. This merits further investigation.

\section{References}

1 Nidorf SM, Fiolet ATL, Mosterd A et al. Colchicine in patients with chronic coronary disease. N Engl J Med 2020;383:1838-47.

2 Herrington W, Lacey B, Sherliker P, Armitage J, Lewington S. Epidemiology of atherosclerosis and the potential to reduce the global burden of atherothrombotic disease. Circ Res 2016;118:535-46.

3 Khan MA, Hashim MJ, Mustafa $\mathrm{H}$ et al. Global epidemiology of ischemic heart disease: Results from the global burden of disease study. Cureus 2020;12:e9349.

4 Roth GA, Johnson C, Abajobir A et al. Global, regional, and national burden of cardiovascular diseases for 10 causes, 1990 to 2015. J Am Coll Cardiol 2017;70:1-25.

5 Libby P, Loscalzo J, Ridker PM et al. Inflammation, immunity, and infection in atherothrombosis: JACC review topic of the week. J Am Coll Cardiol 2018;72:2071-81.

6 Libby P, Okamoto Y, Rocha VZ, Folco E. Inflammation in atherosclerosis: transition from theory to practice. Circ J 2010;74:213-20.

7 Ridker PM, Everett BM, Thuren T et al. Antiinflammatory therapy with canakinumab for atherosclerotic disease. N Engl J Med 2017; 377:1119-31.

8 Nidorf SM, Eikelboom JW, Budgeon CA, Thompson PL. Low-dose colchicine for secondary prevention of cardiovascular disease. J Am Coll Cardiol 2013:61:404-10.

9 Tardif JC, Kouz S, Waters DD et al. Efficacy and safety of low-dose colchicine after myocardial infarction. N Engl J Med 2019;381: 2497-505. 